\title{
Deeplasmid: Deep learning accurately separates plasmids from bacterial chromosomes
}

William B Andreopoulos ${ }^{1,4, *, \&}$, Alexander M Geller,3, Miriam Lucke ${ }^{\star, 3}$, Jan Balewski $^{2}$, Alicia Clum ${ }^{1}$, Natalia Ivanova ${ }^{1}$, and Asaf Levy ${ }^{\&, 3}$

1 Joint Genome Institute, US Department of Energy, LBNL Berkeley, California, USA

2 National Energy Research Scientific Computing Center (NERSC), Berkeley, California, USA

3 Department of Plant Pathology and Microbiology, The Institute of Environmental Science, The Robert H. Smith Faculty of Agriculture, Food, and Environment, The Hebrew University of Jerusalem, Rehovot, Israel

${ }^{4}$ Department of Computer Science, San Jose State University, CA, USA.

* These authors contributed equally

\& Corresponding author.

\begin{abstract}
Plasmids are mobile genetic elements that play a key role in microbial ecology and evolution by mediating horizontal transfer of important genes, such as antimicrobial resistance genes. Many microbial genomes have been sequenced by short read sequencers and have resulted in a mix of contigs that derive from plasmids or chromosomes. New tools that accurately identify plasmids are needed to elucidate new plasmid-borne genes of high biological importance. We have developed Deeplasmid, a deep learning tool for distinguishing plasmids from bacterial chromosomes based on the DNA sequence and its encoded biological data. It requires as input only assembled sequences generated by any sequencing platform and assembly algorithm and its runtime scales linearly with the number of assembled sequences. Deeplasmid achieves an AUC-ROC of over $93 \%$, and it was much more precise than the state-of-the-art methods. Finally, as a proof of concept, we used Deeplasmid to predict new plasmids in the fish pathogen Yersinia ruckeri ATCC 29473 that has no annotated plasmids. Deeplasmid predicted with high reliability that a long assembled contig is part of a plasmid. Using long read sequencing we indeed validated the existence of a $102 \mathrm{Kbp}$ long plasmid, demonstrating Deeplasmid's ability to detect novel plasmids.
\end{abstract}

Availability: The software is available with a BSD license: deeplasmid.sourceforge.io . A Docker container is available on DockerHub under: billandreo/deeplasmid . 


\section{Contact: wandreopoulos@lbl.gov alevy@mail.huji.ac.il}

\section{Introduction}

Plasmids are ubiquitous extrachromosomal elements capable of semi-autonomous replication and transmission between microbial host cells. Typically, bacterial plasmids are small $(<80 \mathrm{~kb})$ circular replicons. Natural plasmids often carry a cargo of "accessory genes" that confer beneficial traits to the microbial host, such as antibacterial resistance $(1,2)$, bacteriophage defense $(3,4)$, heavy metal tolerance $(5)$, virulence $(6,7)$, or unique catabolic pathways (8), thereby improving bacterial adaptation to dynamic environments. Some plasmids carry toxins and antibiotic resistance genes and thereby constitute a serious threat to human health (9). Finally, plasmids are involved in plant-microbe interactions; for instance, the nodulation plasmids of rhizobia guide the symbiosis of bacteria with plants (10). Plasmid transmission by conjugation provides an efficient mechanism of horizontal gene transfer and facilitates the spread of accessory genes in bacterial populations and communities. Therefore, the studies of plasmid genetics, evolution, and dynamics in bacterial populations have many wide-reaching practical applications, such as clinical management of antibiotic resistance (2), development of the industrial strains of bacteria for bioremediation (3) and biofertilization (4). In addition, identification of new plasmids may guide the discovery of novel antibiotic resistance genes, toxins, and genes directly involved in host-microbe interactions, and may be used as new tools for efficient gene cloning and exogenous protein expression.

Advances in genomic sequencing technologies have enabled high-throughput sequencing of genomes of microbial isolates and environmental populations (through metagenome sequencing), including their respective plasmidomes - the total collection of encoded plasmids (5). Identification and classification of plasmid sequences in this treasure trove of genomic and metagenomic data can provide a unique opportunity to study the mechanisms of plasmid persistence, transmission, and host specificity, as well as the flow of accessory genes. However, in silico identification of plasmid contigs in whole-genome shotgun sequences (WGS) is challenging. The challenge derives from poor genome assembly that leads to numerous plasmid-size contigs that are difficult to characterize as derived from plasmid or chromosomes. In addition, there is a limited number of high-quality, completely sequenced reference plasmids that can be compared to while annotating new genomes (6). Sequences from plasmids occasionally integrate into chromosomes, making it difficult to computationally characterize contigs from these chromosomes as plasmids or chromosomes. Further, sometimes plasmid genes have features resembling those of essential chromosomal genes (11).

A variety of in silico methods assisting with separation of plasmid sequences from 
chromosomal contigs have been developed. Some of them target a subset of plasmids mostly of clinical relevance, such as PlasmidFinder/pMLST (12) for detection and typing of plasmids from Enterobacteriaceae and selected Gram-positive strains. Other tools, such as PLACNET (13) rely on a combination of reference genomes and manual curation to restructure an assembly graph and separate putative plasmid contigs from those of chromosomal origin. plasmidSPAdes (14), cBAR (15), PlasFlow (16), Recycler (17), and PlasmidSeeker (18) are fully automated and perform identification of putative plasmid contigs in genome assemblies by analyzing the topology and read coverage of an assembly graph (Recycler and plasmidSPAdes) or DNA composition of assembled contigs (cBar and PlasFlow). Recycler works on paired-end reads and detects circular plasmids by leveraging assembly graphs from conventional assembly tools to assemble circular sequences likely to be plasmids (17). HyAsP starts from raw reads and combines read depth with GC content, as well as reference-based occurrences of known plasmid genes in the assembly (19). An assessment of methods that assemble plasmids from short reads (20) concluded that their accuracy is reliant on a difference in the coverage of plasmids and chromosomes; for some assemblies they demonstrated close to a $90 \%$ precision of plasmid finding with just $55 \%$ recall, whereas for assemblies with $80 \%$ recall generally the false positive rate increases by $20 \%$ (20). Moreover, most of these tools were not tested for their ability to detect novel plasmids that are experimentally validated following the computational prediction.

Existing tools have limitations due to their reliance on the circularity of the topology, bias towards certain taxonomies used in training (e.g. in PlasmidFinder) and coverage of a de Bruijn assembly graph constructed from k-mers found in reads (e. g. Recycler and plasmidSPAdes). The two software packages: cBAR (15) and PlasFlow (16), satisfy the above criteria, since they utilize only two types of data: assembled sequences themselves (PlasFlow) and features extracted from assembled sequences (cBar). PlasFlow relies on a deep neural network to find hidden structures encoded in the assembled sequences, while cBar finds plasmids by applying self-organizing maps (SOMs) to the extracted features in the form of pentamer profiles of contigs and scaffolds. The two methods also differ in the way their models are trained: PlasFlow was developed as a tool for finding plasmids in metagenome data and is pre-trained on sequence fragments of up to $10 \mathrm{~kb}$ long, since metagenome assemblies are typically very fragmented. In contrast, cBAR's model is based on the pentanucleotide profiles of full-length sequences of known plasmids and chromosomes. While both cBAR and especially PlasFlow demonstrated superior performance in comparison to other methods of plasmid identification (16).

Our goal was to develop a tool for post-assembly identification of complete plasmids and plasmid-derived contigs, which (i) has high accuracy, (ii) is not biased towards the sequences of certain topology or taxonomic origin, and (iii) is able to run on genome assemblies from either short-read or long-read sequencing technologies without assembly graph or coverage information. We also confirmed that inclusion of genes and 
other functional features in addition to DNA sequence composition is helpful for contig and scaffold classification. We present a new Deep Learning (DL)-based method, Deeplasmid, for identification of plasmid contigs and scaffolds in WGS assemblies of microbial isolate genomes, which achieves an AUC-ROC of $93 \%$ on a sixfold crossvalidation. Our method relies on a combination of assembled sequences and extracted features, including GC content (21), oligonucleotide composition, hits to plasmid- or chromosome-specific genes, as well as gene density within the contig. Since it does not require raw read data, assembly graph or coverage information, it can be applied to assembled WGS data, including shotgun metagenomic data, generated by any sequencing platform and assembly algorithm. We describe our Deeplasmid model, the training and testing methodology, and show that it is capable of automated detection of plasmid sequences with over $94 \%$ accuracy. Deeplasmid surpasses the accuracy of other tools largely due to its use of discriminating gene and protein features. We compare the accuracy of our trained model on large plasmid-containing microbial test datasets against the alternative tools cBAR and PlasFlow, thus achieving a better precision and comparable recall. Finally, we used a whole genome sequencing project of a specific microbe, Yersinia ruckeri ATCC 29473, applied Deeplasmid and predicted a novel plasmid in this strain. We then performed a sequencing experiment to validate that the new plasmid indeed exists as a separate replication unit. This led to discovery of a new plasmid in this pathogenic strain.

\begin{tabular}{|l|l|l|}
\hline Name & Definition & Type \\
\hline gc_content & GC content of contig & Float [0-1] \\
\hline A(C/G/T)_longest_homopolymer & Length of longest homopolymer & Integer \\
\hline A(C/G/T)_total_homopolymer & $\begin{array}{l}\text { Total number of homopolymers of } \\
\text { length }>5\end{array}$ & Integer \\
\hline hit_chromosome_proteins & Hit to chromosome proteins & Boolean 0/1 \\
\hline hit_plasmid_proteins & Hit to plasmid proteins & Boolean 0/1 \\
\hline hit_plasmid_ORls & Hit to plasmid ORI & Boolean 0/1 \\
\hline gene_count & Number of genes in scaffold & Integer \\
\hline gene_percent & Coding percent of scaffold & Float [0-1] \\
\hline polypeptide_aa_avg_len & $\begin{array}{l}\text { Average length of amino acid } \\
\text { sequence }\end{array}$ & Integer \\
\hline len_sequence & Scaffold seq length & Integer \\
\hline
\end{tabular}


Table 1. Definition of 16 features per sequence

\section{Materials and Methods}

\section{ACLAME-RefSeq training dataset}

We prepared the labeled dataset based on two sources. As negative instances we used 50 different genera from the RefSeq.microbial dataset (22), from which plasmid and any mitochondrial or chloroplast sequences were removed based on their fasta header names. The training included Archaeal chromosomal sequences from 40 genera, which are found in RefSeq.archaea. As positive instances we used the ACLAME dataset (23), which contains 1,056 fully-sequenced plasmids that were manually curated by experts. ACLAME has higher-quality curation than refseq.plasmids since some of the NCBI records tagged as plasmids are mislabeled as chromosomal sequences and many entries do not represent complete records or contain sequence fragments of unknown origin (24, 25). From ACLAME we discarded 39 sequences (3.69\%) that were shorter than $1 \mathrm{kbp}$ or longer than $330 \mathrm{kbp}$ because the scaffolds and contigs longer than $330 \mathrm{~kb}$ are almost invariably chromosomes or megaplasmids or chromids (genetic elements with plasmidtype replication systems, but carrying some indispensable genes (11)). We did not deal with the last two classes as they are special cases. To balance the dataset size we randomly selected 40,000 sequences from the RefSeq.microbial dataset, which is two orders of magnitude larger than ACLAME.plasmid dataset of size 1,017. The imbalance $1 / 40$ in the sequence count in our ACLAME-RefSeq training dataset was compensated during the training by oversampling the ACLAME dataset. Data were shuffled before training.

\section{Input format}

A single training data element consists of the label and two input words: $x_{\text {seq }}$ - a $300 \mathrm{bp}$ contiguous subsequence sampled randomly from the full original scaffold sequence and $\mathrm{x}_{\mathrm{f}}$ - a vector containing 16 features extracted from the full sequence, as described in Table 1. In order to ensure feature values like gene count, gene coding percentage, or sequence length are meaningful, the features are computed on the entire scaffold, and the values are copied into the $x_{f}$ feature vectors for all 300bp sequences subsampled from the scaffold. The number $(\mathrm{m})$ of $300 \mathrm{bp}$ subsequences sampled from each scaffold is proportional to the square root of the scaffold length. The number of samples per scaffold was chosen according to $m=10+$ sqrt(seq_len/20) to ensure a fair representation of smaller and larger scaffolds, such that longer scaffolds do not overwhelm the training step. Each sample is a different $x_{\text {seq }}$ associated with the feature vector $x_{f}$ from the originating sequence. $x_{\text {seq }}$ is one-hot encoded in 4 nucleotide bases. Namely, it is transformed into a binary array of size $300 \times 4$. The ' $N$ '-base (unknown) is encoded as four 
zeros. The values of $x_{f}$ were normalized to be bound within $[-1,1]$.

\section{Input feature selection}

We initially explored the predictive power of several extracted features in conjunction with existing Machine Learning tools. The particular choice of $x_{f}$ variables shown in Table 1 was based on an initial sensitivity analysis with the Gradient Boosting Classifier, a classic ML method that produces a prediction model in the form of a mixture of decision trees. Moreover, we confirmed the relative importance of a feature by training our tool and running predictions with null values for the feature (namely, excluding the feature) and checking the impact on the error rate (as discussed in the Supp. Info).

We additionally included in $x_{f}$ three plasmid-specific and chromosome-specific features. These features are boolean ( 0 or 1 ) and indicate whether any hit is found to these sets of plasmid or chromosome-specific sequences:

1. Plasmid-specific DNA motifs: these are the origins of replication of known plasmids (26).

2. Plasmid-specific proteins: these are taken from 2,826 known plasmids listed on 2019 in the European Nucleotide Archive: https://www.ebi.ac.uk/genomes/plasmid.html, and removed any plasmids not isolated from Proteobacteria, Firmicutes, Bacteroidetes, or Actinobacteria using NCBI batch entrez function. We only kept plasmids from these four phyla as these are the most commonly sequenced and studied bacterial phyla and as a result most contigs that will be classified by our tool belong to these phyla. Some plasmidic proteins were extracted from publications (27-36) (Table S1). The final list included 136,638, 24,607, 1,163, and 15,449 plasmidic genes from Proteobacteria, Firmicutes, Bacteroidetes, and Actinobacteria respectively.

3. Chromosome-specific proteins: these are based on COGs of genes that are usually carried on chromosomes. The 61 COGs used for making this list are based on chromosomal housekeeping genes that are unclonable in high copy plasmids $(37,38)$. They appear in Table S1.

To reduce sequence redundancy the chromosomal and plasmid proteins were clustered by $90 \%$ identity using cd-hit with otherwise default parameters giving a representative sequence from each cluster (39).

\section{Output format}

A neural network is a function

$$
F\left(x_{\text {seq }}, x_{f} \mid \theta\right)=y .
$$

that accepts sequences of nucleotides xseq and the feature vector $\mathrm{X}_{\mathrm{f}}$. The function $\mathrm{F}$ also depends implicitly on the DL model parameters $\theta$, which are determined during the 
training process. The output of the network, Deeplasmid score y, is computed using the softmax function, which ensures that y satisfies y $\square[0,1]$. By convention, the higher the score is for the sequence, the more likely it is to be a true plasmid.

\section{Model training}

The model was trained with a binary cross-entropy loss function (40) and Adam optimizer (41). We performed supervised learning on the balanced set of $6 \times 10^{5}$ data points with a batch size of 200 . The initial learning rate was set to 0.001 . Typically we sample $50-100$ 300bp long sequences per scaffold.

We used the $k$-fold cross-validation method, setting $k=6$, with five data segments merged as the 'training' set and one validation segment that provided the loss (model error) as feedback during training. The 'test' data set was hidden during the training. We performed 6 independent trainings, cycling the segments to allow each of six segments to influence a different model $\theta_{\mathrm{k}}$. Figure 1 illustrates the k-fold training method. Each model was trained for 30 epochs, until it converged, as shown in Figure 2a.

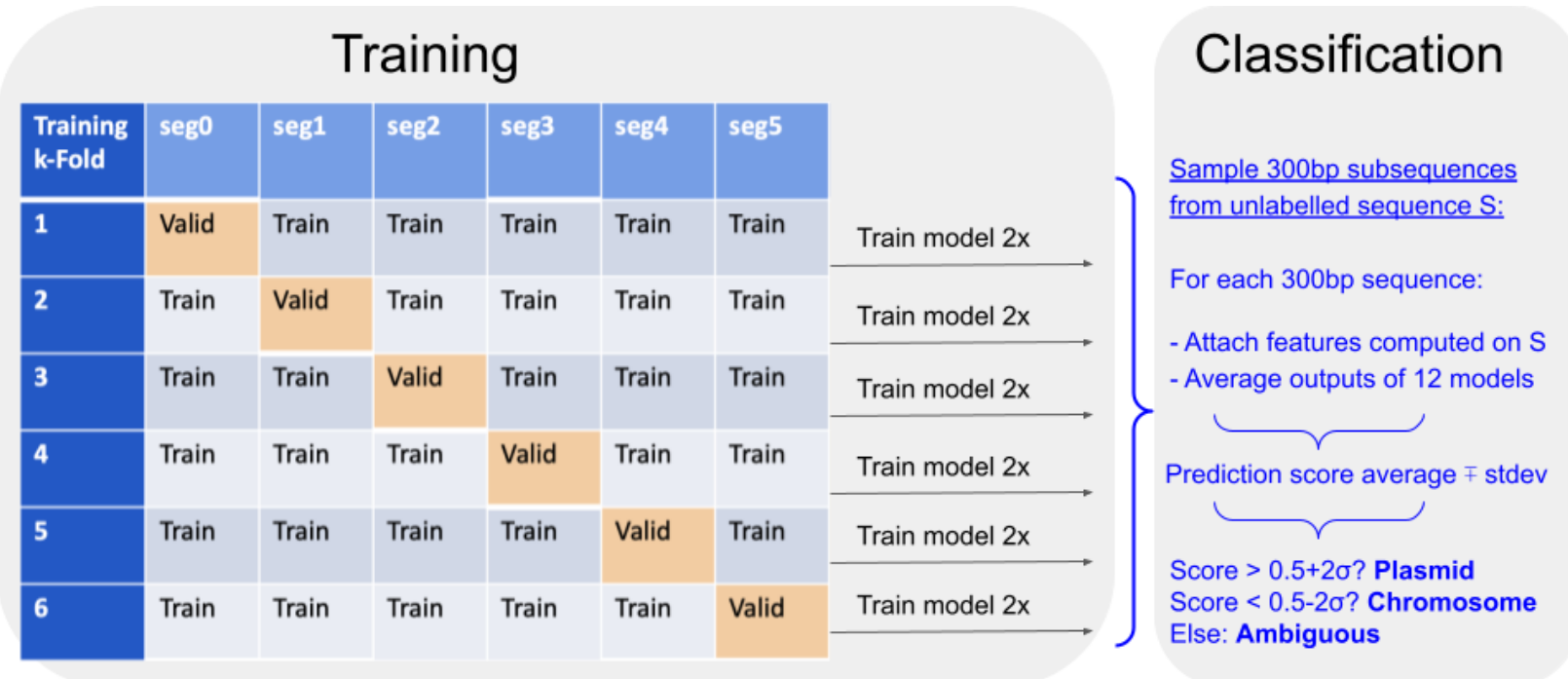

Fig. 1. Deeplasmid training, validation and testing. The plasmid and chromosome dataset was split into six segments, of which five were used in training a model. The $6^{\text {th }}$ segment was used for validation of the trained model. We repeated over the training twice to derive 12 different models. Using 12 models allows reducing the effects of random variance in the predictions.

\section{Model topology}

We have used the LSTM-based network (42) (Figure S4) to transform the one-hot 
encoded nucleotide sequence into a one-dimensional vector. The left branch is made out of two LSTMs, accepts a 300bp nucleotide sequence $\mathrm{x}_{\text {seq }}$, and compresses information into a vector of 40 features. The right branch is fully connected, accepts the feature vector $x_{f}$, and produces a vector of 20 features. Both outputs are concatenated and passed to another block of fully connected layers whose output is one value - the Deeplasmid score y (Eq. 1). This model was implemented in Keras (43) with Tensorflow 1.3.0 (44) as the backend. The deep learning model architecture is shown in Figure S3.

(a)
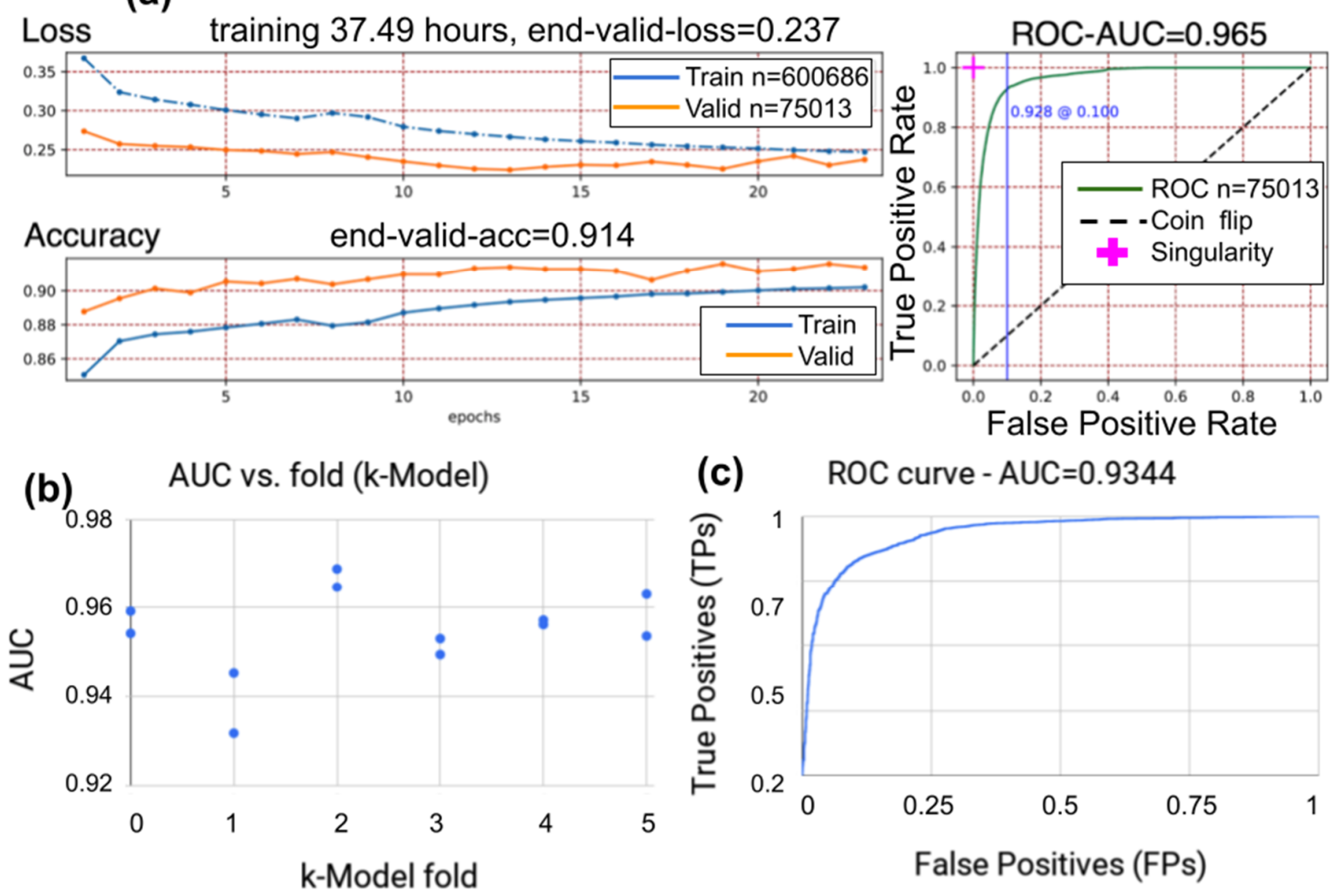

(c) ROC curve - AUC $=0.9344$

Fig. 2. (a) Training convergence through the epochs. Loss and accuracy are shown as a function of epochs. The training and validation data are balanced by oversampling the plasmid class. (b) The training was repeated 12 times on the plasmid-chromosome dataset to derive twelve models (two per validation segment). All models achieved an accuracy (AUC) on the validation segment of over 0.93 with a small statistical variance in the prediction accuracy. (c) The ROC-AUC on the IMG test dataset after training the model is 0.9344 .

\section{Prediction for one 300bp sequence}


For each k-fold we saved two models, resulting in 12 different saved models. This was done to reduce the effects of random variance in the predictions, as well as to ensure that the results were reproducible for each $\mathrm{k}$-fold. To make a prediction on a $300 \mathrm{bp}$ sequence we ran the sequence through all 12 models and then average the score:

The results are shown in Table S2.

$$
\hat{y}=\sum_{i=1}^{12} \quad y(\theta i) / 12 .
$$

\section{Prediction for one scaffold}

One scaffold is sampled 50-100 times and for each 300bp sequence the average score is computed as in (Eq. 2). Next, the scaffold-average score (yavr) and its standard deviation $(\sigma)$ are computed. We allow for 3-way classification as "plasmid", "chromosome", or "ambiguous":

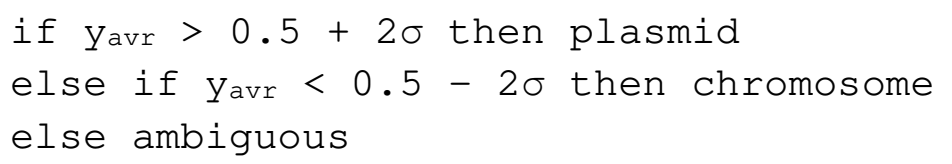

\section{DNA extraction and Oxford Nanopore sequencing}

We validated our plasmid computational prediction using genome re-sequencing with Oxford Nanopore long read sequencing. We used strain Yersinia ruckeri ATCC 29473 (IMG genome ID: 2609460118), which was received as a gift from Dr. Yasuo Yoshikuni. Bacteria grew in the final volume of $2 \mathrm{~L}$ Luria Broth until OD 6000.3 was reached. DNA was extracted with Qiagen Genomic Tip 100/G (Cat No./ID: 10243) and Genomic DNA Buffer Set (Qiagen, Cat No./ID: 19060), by following suggested instructions. DNA concentration and quality were tested with Nanopore, Qubit and TapeStation. Prior sequencing samples were prepared with Ligation Sequencing Kit (SQK-LSK109) and Native Barcoding Expansion 1-12 (EXP-NBD104) and finally sequenced by Oxford Nanopore MinION. Reads were assembled using Canu 2.0 (45). Reads were also assembled in parallel by Shasta 0.6 .0 (46). The 3,754,417 bp circular DNA and the 102,560 circular DNA were found from the Canu and Shasta assemblies, respectively. To map linear scaffolds of Yersinia ruckeri ATCC 29473 to the newly assembled plasmids, blastn was used, with a bitscore cutoff of 30,000. The blastn hits were visualized using DNAFeaturesViewer (47). The IMG scaffold names were shortened in Figure 4; all scaffolds displayed are prefixed by "Ga0059170_", e.g. scaffold 114 is named "Ga0059170_114" in the IMG database. Scaffold "Ga0059170_103" coordinates 1145,000 were mapped to the newly found plasmid (fragment is at 8 o'clock in Figure 4, marked $103^{\star}$ ), away from the rest of the scaffold (12 to 5 o'clock in Figure 4). This 
subsequence is small enough to be processed by Deeplasmid and was thereby re-ran through Deeplasmid to be predicted as a separate piece of DNA. Annotation (Figure 4C) was performed based on IMG scaffold annotations (scaffolds Ga0059170_112 and Ga0059170_113).

\section{$\underline{\text { Results }}$}

\section{Training and feature selection}

We assembled a training dataset of bacterial and archaeal chromosomes from 90 genera that was retrieved from Refseq, and we retrieved 1,017 plasmids from ACLAME, (Materials and Methods). Features included sequence-related physical features and features related to the genetic content of the contig (Table 1, Table S1, Materials and Methods). The first feature group includes gc_content, repeats: number and size of the longest homopolymers, and total length of the sequence. The second feature group includes boolean variables of whether the sampled sequence shares similarity with chromosomal genes, plasmidic genes, or origin of replication sequences. For example, ribosomal proteins are usually carried on chromosomes and plasmid replication and mobilization genes are carried on plasmids. We also included in this group the number of genes carried on the scaffold, the percent of the scaffold which is coding, and the size of the genes ("polypeptide_aa_avg_len") as through manual inspection we noted that plasmids tend to have more intergenic regions and smaller genes than chromosomes.

\section{Deeplasmid predictions for the 'test' data}

The training dataset was divided into six segments (Materials and Methods). One segment, called 'validation', was used for validating a model's training. Twelve models were trained in total, two for each selection of a validation segment. The model training over 30 epochs is characterized by an increase in prediction accuracy (defined as the ratio of correct classifications to all queries) and decrease in error (loss) on the validation segment. The Receiver Operating Characteristic (ROC) curve is shown in Fig. 2a. The Area Under the Curve (AUC) for Deeplasmid reached 0.93. To be consistent with the standard definition of AUC we have forced predictions to be binary by reducing the standard deviation $\sigma$ to 0 and setting threshold $\theta$ to 0.5 . The predictions made by Deeplasmid are averaged over 12 models (Materials and Methods). Fig. 2b shows prediction accuracy individually for each of the 12 models over the 'validation' data segment. There is a high agreement between models.

\section{Feature significance}


We also evaluated the significance of various sets of features by calculating the decrease in average AUC of the 12 models on the training data set after setting the features to zero values (thus knocking them out). We retrained the model on various combinations of features. Most of the runs where features were removed resulted in a mean AUC drop more than 3 standard deviations away from the mean AUC achieved when using all features. The use of all features resulted in a mean AUC of 0.897 with a relatively small standard deviation (over 12 runs) of 0.0026 . The mean AUC dropped by $5 \%$ to 0.847 after removing the hits to plasmid and chromosome-specific genes demonstrating the significance of this feature in classification of annotated contigs. When using only hits to plasmid and chromosome-specific genes (in addition to the sequence data) the mean AUC also dropped to 0.8548 . Other features, each one separately, such as sequence length, homopolymer-related features (the longest homopolymer and the total number of homopolymers of length $>5$ ), and gene density in the scaffold had relatively little contribution. However, we trained the model with all features since removing features translated to an increase in the error of prediction. We provide an analysis of the mean AUCs observed over 12 models with various feature sets (Table S3). We conclude that using curated biological information provides a clear advantage over previous algorithms, which only used "physical" features of the sequences (such as gc content and scaffold length).

\section{Testing Deeplasmid model on independent dataset: isolate genomes from IMG database}

To test Deeplasmid, we used an independent dataset retrieved from IMG database (48, 49). We downloaded the sequences of 1,834 isolate genomes that have at least one replicon annotated as plasmid. This set included a total of 6820 scaffolds and contigs, with 3093 of them annotated as plasmids and 3727 annotated as chromosomes. Figure $2 \mathrm{C}$ shows the ROC curve for the IMG test dataset, achieving an AUC of 0.9344. This suggests that the trained Deeplasmid model is applicable widely, and does not suffer from overfitting. Figure 3 shows the counts of plasmid and chromosomal scaffolds assigned a certain score by Deeplasmid. Setting the threshold for separating the two classes at 0.5 , the precision or purity of the predicted positive class (plasmid sequences) is $94 \%$. On the other hand, recall is $77 \%$ (details in Suppl. Info) indicating that the DL model missed some plasmids, classifying them as chromosomal fragments.

Similar to our training methodology, the prediction was done on scaffolds of length $1 \mathrm{~K}$ $330 \mathrm{~K}$ bases, while scaffolds outside this length range were classified as either too long or too short. There are also 63 ambiguous predictions, which are split between the chromosomal and plasmid classes. 


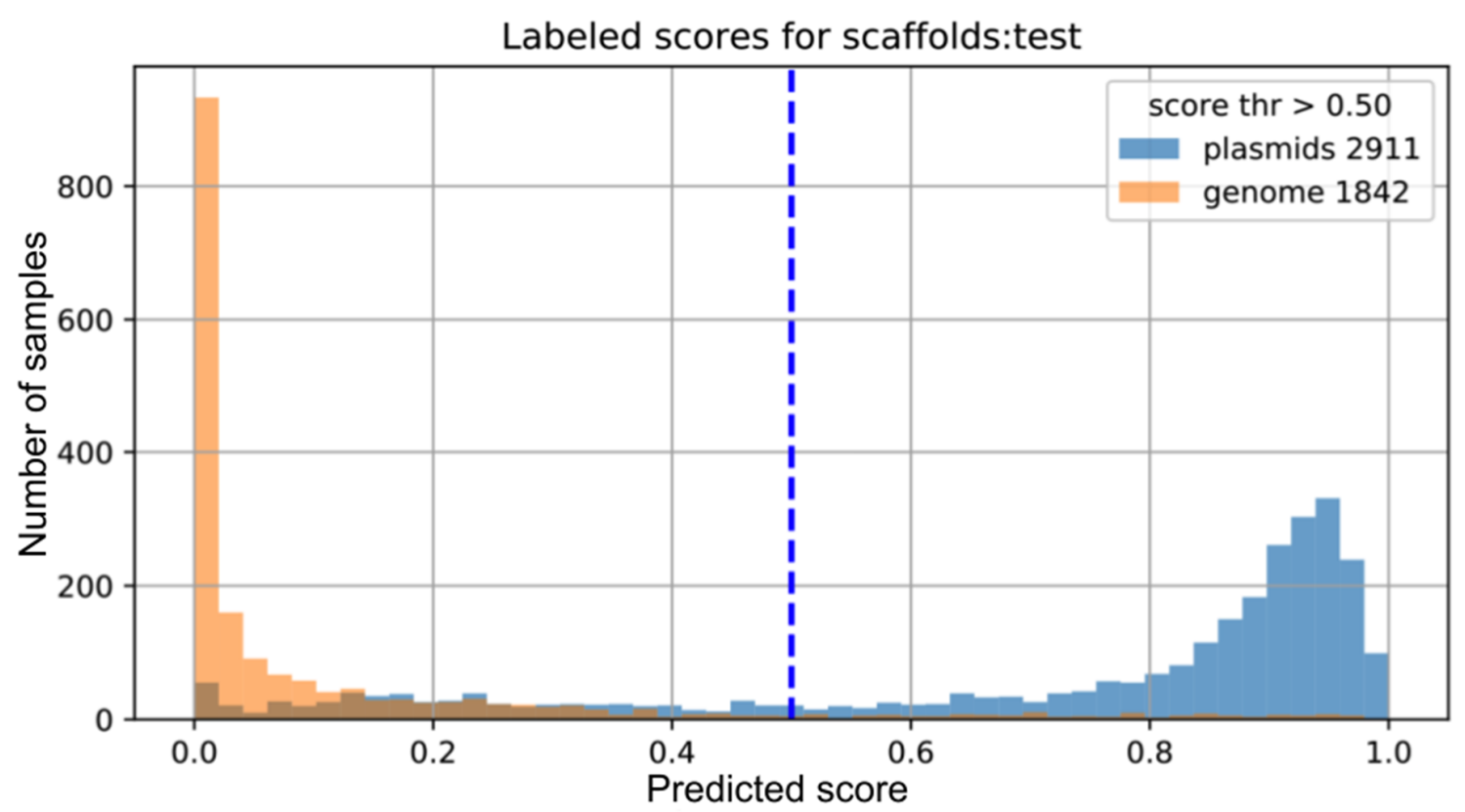

Fig. 3. Evaluation on the IMG test dataset. The class separation is clear based on a threshold of 0.5. The percent of plasmids classified above the threshold is $2264 /(2264+599+48)=77.77 \%$ (recall). The percent of chromosomes classified below the threshold is $1702 /(1702+140)=92.4 \%$.

Next, we used this dataset to compare the performance of our $\mathrm{DL}$ model to the comparable state-of-the-art programs PlasFlow [16] and cBar [15] (Table 2). Deeplasmid achieved a precision of 0.94 on this dataset (compared to 0.68 for both of the other tools), with a recall comparable to PlasFlow and cBar. Overall, the F1-score, defined as 2.P.R/(P+R), for Deeplasmid was 0.85 , which is higher than either PlasFlow $(0.69)$ or cBar (0.73). This comparison demonstrates that addition of biological features vastly improves the performance of programs with otherwise similar inputs and goals.

\begin{tabular}{|l|l|l|l|l|l|l|l|l|l|l|l|l|l|l|l|}
\hline & \multicolumn{4}{|l|}{$\begin{array}{l}\text { Classified as } \\
\text { Plasmid }\end{array}$} & \multicolumn{4}{l|}{$\begin{array}{l}\text { Classified as } \\
\text { Chromosome }\end{array}$} & \multicolumn{3}{l|}{$\begin{array}{l}\text { Classified } \\
\text { Ambiguous }\end{array}$} & \multicolumn{2}{l|}{$\begin{array}{l}\text { lrec } \\
\text { (TP/TP+FP) }\end{array}$} & \multicolumn{2}{l|}{$\begin{array}{l}\text { Rec } \\
\text { (TP/TP+FN) }\end{array}$} \\
\hline Truth & $\boldsymbol{D}$ & $\boldsymbol{P}$ & $\boldsymbol{C}$ & $\boldsymbol{D}$ & $\boldsymbol{P}$ & $\boldsymbol{C}$ & $\boldsymbol{D}$ & $\boldsymbol{P}$ & $\boldsymbol{C}$ & $\boldsymbol{D}$ & $\boldsymbol{P}$ & $\boldsymbol{C}$ & $\boldsymbol{D}$ & $\boldsymbol{P}$ & $\boldsymbol{C}$ \\
\hline Plasmid & 2264 & 2213 & 2519 & 599 & 229 & 581 & 48 & 658 & 0 & 0.94 & 0.68 & 0.68 & 0.77 & 0.71 & 0.81 \\
\hline Chromo. & 140 & 1046 & 1190 & 1687 & 1722 & 2537 & 15 & 959 & 0 & F1 score: & 0.85 & 0.69 & 0.73 \\
\hline
\end{tabular}

Table 2. Comparison of Deeplasmid (" $D$ ") to PLASFlow (" $P$ ") to cBar (" $C$ ") on the IMG database. With Deeplasmid the purity of the predicted plasmid sequences is $94 \%$ (precision) and $77 \%$ of known plasmids are predicted correctly (recall). 
The runtimes were taken on a Cray XC40 supercomputer (5 Intel Xeon "Haswell" nodes with $120 \mathrm{~GB}, 16$ cores). The training runtime was 12.94 hours for the ACLAME and RefSeq.microbial dataset with $41 \mathrm{~K}$ sequences. For 30 epochs that translates to 26 minutes per epoch. The prediction runtime was $<2$ seconds per scaffold or under two minutes for a microbial genome assembly, assuming a typical microbial genome assembly contains 1-60 scaffolds. This satisfies the scalability requirement for an automated plasmid finding tool, highlighting Deeplasmid's potential for widespread application on large-scale genomic and metagenomic data.

\section{Experimental validation of a new plasmid based on Deeplasmid prediction}

In order to demonstrate Deeplasmid's ability to predict plasmids in biological samples, we focused on the fish pathogen Yersinia ruckeri ATCC 29473 (IMG genome ID: 2609460118). Until this work, this strain had only been sequenced by 454 and Illumina short read sequencers, resulting in 15 linear scaffolds and presumably lacking a plasmid (50). Using comparative genomics, researchers studying a similar bacterial strain inferred that Yersinia ruckeri ATCC 29473 may encode a plasmid, but no long read sequencing was performed to confirm this (51). The 15 linear scaffolds of Yersinia ruckeri ATCC 29473 were then used as input to Deeplasmid, and labeled as either plasmid or chromosomal. One long scaffold of $57 \mathrm{Kbp}$ (IMG scaffold 112, Figure 4A) got a Deeplasmid score of 0.8 strongly suggesting that it is derived from a plasmid. In contrast, other scaffolds were either very long (e.g. IMG scaffold 103 of $1.6 \mathrm{Mbp}$ ) or received Deeplasmid score below 0.3 , suggesting that they derive from a bacterial chromosome. To validate these labels, we grew Y. ruckeri bacteria in the lab, extracted DNA, and sequenced it with a long read sequencing method (Oxford Nanopore Technology). In contrast to the previous short read methods, we were able to find large circular pieces of DNA. We found a $~ 3.7 \mathrm{Mbp}$ chromosome, and a 102 kbp plasmid. Beyond the fact that it is circular, we are confident the latter piece of DNA is a plasmid since it has very high similarity to a known Yersinia plasmid (pYR3; Genbank: LN681230.1).

Upon mapping the linear scaffolds onto the newly sequenced circular DNA fragments, we indeed find that predicted plasmid fragments map to the $102 \mathrm{kbp}$ plasmid, demonstrating the predictive power of Deeplasmid and its ability to detect large plasmids in genomic data (Figure 4A). We note that a number of the linear scaffolds for this genome did not undergo Deeplasmid prediction due to their large size (>330kb). Exactly because of their large size, they are assumed to be chromosomal in origin. However, those within the size range of Deeplasmid functionality were largely predicted to be chromosomal in origin, along with some misclassifications (Figure 4).

Looking at the functions of the genes carried on the plasmid, we see many genes 
previously found on similar plasmids (51), and that have plasmid-related functions. We identified Type IV pilus genes, which may be used for transfer of plasmid from one cell to another (52), or possibly used as a virulence factor (51). Also encoded on the plasmid is the Type IV Secretion System, which may also be involved in plasmid transfer (53) and/or virulence (51). We also detected on the plasmid is RelE, a toxin which is commonly found in plasmid addiction systems (54). Furthermore, we found mobilization genes like transposon genes, integrases, and DNA recombinases. Overall, we conclude that this is a bona fide plasmid based on its circularity, separation from the main chromosome, similarity to known plasmids, and plasmidic gene content.

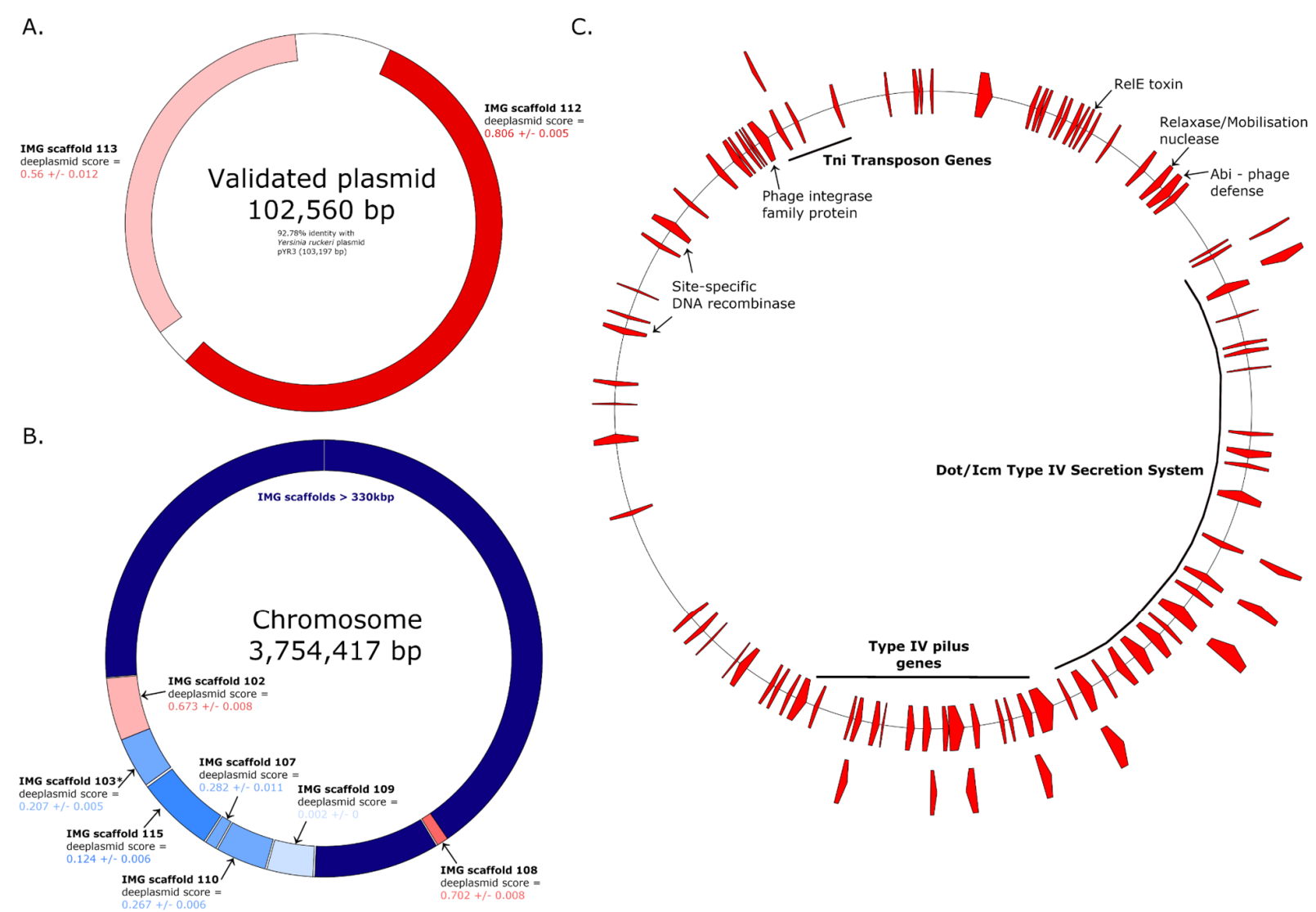

Figure 4. Deeplasmid validation. Yersinia ruckeri ATCC 29473 was sequenced with Oxford Nanopore MinION and assembled with Canu and with Shasta. (A) The assembled contigs included a circular piece of DNA that shares $92.78 \%$ identity with a known Yersinia plasmid (pYR3; Genbank: LN681230.1). Two linear scaffolds of this genome were predicted by Deeplasmid to be from plasmids (shades of red), and indeed they align with the newly-found plasmid. (B) The assembled contigs also contained a chromosome. Most of the linear scaffolds for this genome did not undergo Deeplasmid prediction, due to their large size (>330kb; dark navy blue). However, those within the size range were largely predicted to be chromosomal in origin (shades of blue), along with two 
misclassifications (shades of red). Scaffold $103^{*}$ is a subsequence of a larger IMG scaffold; this short region was predicted by Deeplasmid here (see Materials and Methods). (C) Functions of many ORFs identified on the validated plasmid are classically associated with plasmids.

\section{Discussion and Conclusion}

In this work we provide an accurate algorithm to classify assembled contigs or scaffolds generated by any sequencing platform and assembly algorithm, as parts of plasmids and chromosomes using a deep learning approach. By training deep learning models on the specific features of plasmids and chromosomes, we have shown that it is possible to efficiently separate plasmids from chromosomal sequences. While physical sequence features can be used to predict if a sequence is of plasmid or chromosome origin, the DNA sequence itself improves the deep learning models by keeping a memory of what came earlier in the sequence. The reason is that statistical features of sequence composition, such as GC content or oligonucleotide profiles, fail to capture the nucleotide composition over the length of the sequence, as explained in earlier work (8). One of the reasons why our Deeplasmid model has better precision at predicting plasmids than other methods is that it averages predictions over multiple 300bp windows sampled over the length of the sequence, instead of analyzing contigs and scaffolds as a single DNA molecule. As a result, each prediction for a 300bp sequence contributes to the overall result. Additionally, we complemented sequences with extracted feature data that enhance the prediction accuracy. The plasmid-specific ORIs, chromosome-specific genes and GC content serve as essential data features. Averaging the predictions over many 300-base sequences and including biologically meaningful features resulted in a better ability to classify a sequence as plasmidic or chromosomal.

Just like other methods that rely on the nucleotide composition signatures of plasmids and chromosomes, whether as hidden or extracted features, Deeplasmid is likely to have problems with very short sequences for which it may have trouble obtaining a proper sequence signature. For this reason we limited the sequences used in the model construction to those of minimum $1 \mathrm{~Kb}$ length. There are also very few existing plasmids that are larger than $330 \mathrm{Kbp}$ and therefore we could not train our algorithm on these megaplasmids.

Prediction of plasmids is complicated in large genomic datasets with possible chromosomal integrations of plasmids. Current challenges in the field of plasmid identification include discriminating chromosomes that resemble plasmid sequences or plasmids with chromosomal replication genes. Another challenge is to achieve high prediction accuracy on unknown or understudied microbial lineages that may contain 
exotic plasmids, since any machine learning tool will be trained with the current knowledge.

Deeplasmid predicts plasmids with a low false positive error rate using only an assembled fasta file as input. It can identify both circular and linear plasmids. The output is a perscaffold classification of chromosomal, plasmid, or ambiguous contig, along with a score representing the confidence of the prediction. Although the default probability threshold for separation of classes was set at 0.5 based on our benchmarking, users can specify their own filtering cutoffs. Deeplasmid out-performed other available tools in terms of accuracy for single microbial assembly plasmidome analyses. Moreover, we provided experimental evidence for a new plasmid that was predicted using Deeplasmid. A future research direction is to employ Deeplasmid for large-scale identification of plasmids in large-scale metagenomic data from different environments or to uncover novel plasmidborne antimicrobial resistance genes or novel microbial genes that are horizontally transferred via plasmids. This and myriad other high-impact applications of Deeplasmid are possible due to its fast running time and scalability.

\section{Supplementary Information}

The Supplementary Information file includes Deeplasmid running instructions, comments on features used in chromosome/plasmid classification, Code Repository, Software Design, Deep learning model architecture, AUC per fold on the training dataset, ROC curve for the IMG test dataset, and others.

\section{Acknowledgements}

We thank NERSC for providing us with high-performance computing support throughout this study. This research used resources of the National Energy Research Scientific Computing Center (NERSC), a U.S. Department of Energy Office of Science User Facility operated under Contract No. DE-AC02-05CH11231. The work conducted by the U.S. Department of Energy Joint Genome Institute, a DOE Office of Science User Facility, is supported by the Office of Science of the U.S. Department of Energy under Contract No. DE-AC02-05CH11231.

$A L$ is generously supported by the Israeli Science Foundation (Grants \#1535/20, \#3300/20), Alon Fellowship of the Israeli council of higher education, The Hebrew University - University of Illinois Urbana-Champaign seed grant, the Israeli Ministry of Agriculture (Grant 12-12-0002), and ICA in Israel. AMG is generously supported by the Kaete Klausner Scholarship and was supported by a scholarship from the Israeli Ministry of Aliyah and Integration. WBA was supported by SJSU's Faculty startup fund.

We thank Dr. Yasuo Yoshikuni for providing us with Yersinia ruckeri ATCC 29473 strain. 
1. Tran,J.H. and Jacoby,G.A. (2002) Mechanism of plasmid-mediated quinolone resistance. Proceedings of the National Academy of Sciences, 99, 5638-5642.

2. Martínez-Martínez,L., Pascual,A. and Jacoby,G.A. (1998) Quinolone resistance from a transferable plasmid. Lancet, 351, 797-799.

3. Klaenhammer,T.R. (1987) Plasmid-directed mechanisms for bacteriophage defense in lactic streptococci. FEMS Microbiol. Rev., 3, 313-325.

4. Sing,W.D. and Klaenhammer,T.R. (1990) Characteristics of phage abortion conferred in lactococci by the conjugal plasmid pTR2030. Microbiology, 136, 1807-1815.

5. Silver,S. and Misra,T.K. (1988) Plasmid-mediated heavy metal resistances. Annu. Rev. Microbiol., 42, 717-743.

6. Baldini,M.M., Kaper,J.B., Levine,M.M., Candy,D.C. and Moon,H.W. (1983) Plasmidmediated adhesion in enteropathogenic Escherichia coli. J. Pediatr. Gastroenterol. Nutr., 2, 534-538.

7. Watson,B., Currier,T.C., Gordon,M.P., Chilton,M.D. and Nester,E.W. (1975) Plasmid required for virulence of Agrobacterium tumefaciens. J. Bacteriol., 123, 255-264.

8. Eaton,R.W. and Timmis,K.N. (1986) Characterization of a plasmid-specified pathway for catabolism of isopropylbenzene in Pseudomonas putida RE204. J. Bacteriol., 168, 123-131.

9. Li,J., Adams,V., Bannam,T.L., Miyamoto,K., Garcia,J.P., Uzal,F.A., Rood,J.I. and McClane,B.A. (2013) Toxin plasmids of Clostridium perfringens. Microbiol. Mol. Biol. Rev., 77, 208-233.

10. Heron,D.S. and Pueppke,S.G. (1984) Mode of infection, nodulation specificity, and indigenous plasmids of 11 fast-growing Rhizobium japonicum strains. J. Bacteriol., 160, 1061-1066.

11. Harrison,P.W., Lower,R.P.J., Kim,N.K.D. and Young,J.P.W. (2010) Introducing the bacterial 'chromid': not a chromosome, not a plasmid. Trends Microbiol., 18, 141148.

12. Carattoli,A., Zankari,E., García-Fernández,A., Voldby Larsen,M., Lund,O., Villa,L., Møller Aarestrup,F. and Hasman,H. (2014) In silico detection and typing of plasmids using PlasmidFinder and plasmid multilocus sequence typing. Antimicrob. Agents Chemother., 58, 3895-3903.

13. Vielva,L., de Toro,M., Lanza,V.F. and de la Cruz,F. (2017) PLACNETw: a webbased tool for plasmid reconstruction from bacterial genomes. Bioinformatics, 33, 3796-3798. 
14. Antipov,D., Hartwick,N., Shen,M., Raiko,M., Lapidus,A. and Pevzner,P.A. (2016) plasmidSPAdes: assembling plasmids from whole genome sequencing data. Bioinformatics, 32, 3380-3387.

15. Zhou,F. and Xu,Y. (2010) cBar: a computer program to distinguish plasmid-derived from chromosome-derived sequence fragments in metagenomics data. Bioinformatics, 26, 2051-2052.

16. Krawczyk,P.S., Lipinski,L. and Dziembowski,A. (2018) PlasFlow: predicting plasmid sequences in metagenomic data using genome signatures. Nucleic Acids Res., 46, e35.

17. Rozov,R., Brown Kav,A., Bogumil,D., Shterzer,N., Halperin,E., Mizrahi,I. and Shamir,R. (2017) Recycler: an algorithm for detecting plasmids from de novo assembly graphs. Bioinformatics, 33, 475-482.

18. Roosaare,M., Puustusmaa,M., Möls,M., Vaher,M. and Remm,M. (2018) PlasmidSeeker: identification of known plasmids from bacterial whole genome sequencing reads. PeerJ, 6, e4588.

19. Müller,R. and Chauve,C. (2019) HyAsP, a greedy tool for plasmids identification. Bioinformatics, 35, 4436-4439.

20. Arredondo-Alonso,S., Willems,R.J., van Schaik,W. and Schürch,A.C. (2017) On the (im)possibility of reconstructing plasmids from whole-genome short-read sequencing data. Microb Genom, 3, e000128.

21. Rocha,E.P.C. and Danchin,A. (2002) Base composition bias might result from competition for metabolic resources. Trends Genet., 18, 291-294.

22. Pruitt,K.D., Tatusova,T. and Maglott,D.R. (2007) NCBI reference sequences (RefSeq): a curated non-redundant sequence database of genomes, transcripts and proteins. Nucleic Acids Res., 35, D61-5.

23. Leplae,R., Lima-Mendez,G. and Toussaint,A. (2010) ACLAME: a CLAssification of Mobile genetic Elements, update 2010. Nucleic Acids Res., 38, D57-61.

24. Galata,V., Fehlmann,T., Backes,C. and Keller,A. (2019) PLSDB: a resource of complete bacterial plasmids. Nucleic Acids Res., 47, D195-D202.

25. Orlek,A., Phan,H., Sheppard,A.E., Doumith,M., Ellington,M., Peto,T., Crook,D., Walker,A.S., Woodford,N., Anjum,M.F., et al. (2017) Ordering the mob: Insights into replicon and MOB typing schemes from analysis of a curated dataset of publicly available plasmids. Plasmid, 91, 42-52.

26. Mei,J., Benashski,S. and Firshein,W. (1995) Interactions of the origin of replication (oriV) and initiation proteins (TrfA) of plasmid RK2 with submembrane domains of Escherichia coli. J. Bacteriol., 177, 6766-6772. 
27. Fang,F.C. and Helinski,D.R. (1991) Broad-host-range properties of plasmid RK2: importance of overlapping genes encoding the plasmid replication initiation protein TrfA. J. Bacteriol., 173, 5861-5868.

28. Light,J. and Molin,S. (1983) Post-transcriptional control of expression of the repA gene of plasmid R1 mediated by a small RNA molecule. EMBO J., 2, 93-98.

29. Unterholzner,S.J., Poppenberger,B. and Rozhon,W. (2013) Toxin-antitoxin systems: Biology, identification, and application. Mob. Genet. Elements, 3, e26219.

30. Gerdes,K., Møller-Jensen,J. and Bugge Jensen,R. (2000) Plasmid and chromosome partitioning: surprises from phylogeny. Mol. Microbiol., 37, 455-466.

31. Petrova,V., Chitteni-Pattu,S., Drees,J.C., Inman,R.B. and Cox,M.M. (2009) An SOS inhibitor that binds to free RecA protein: the PsiB protein. Mol. Cell, 36, 121-130.

32. Zatyka,M. and Thomas,C.M. (1998) Control of genes for conjugative transfer of plasmids and other mobile elements. FEMS Microbiol. Rev., 21, 291-319.

33. Guynet,C., Cuevas,A., Moncalián,G. and de la Cruz,F. (2011) The stb operon balances the requirements for vegetative stability and conjugative transfer of plasmid R388. PLoS Genet., 7, e1002073.

34. Wang,P., Zhu,Y., Zhang,Y., Zhang,C., Xu,J., Deng,Y., Peng,D., Ruan,L. and Sun,M. (2016) Mob/oriT, a mobilizable site-specific recombination system for unmarked genetic manipulation in Bacillus thuringiensis and Bacillus cereus. Microb. Cell Fact., 15, 108.

35. Garcillán-Barcia,M.P., Francia,M.V. and de la Cruz,F. (2009) The diversity of conjugative relaxases and its application in plasmid classification. FEMS Microbiol. Rev., 33, 657-687.

36. Smillie,C., Garcillán-Barcia,M.P., Francia,M.V., Rocha,E.P.C. and de la Cruz,F. (2010) Mobility of plasmids. Microbiol. Mol. Biol. Rev., 74, 434-452.

37. Sorek,R., Zhu,Y., Creevey,C.J., Francino,M.P., Bork,P. and Rubin,E.M. (2007) Genome-wide experimental determination of barriers to horizontal gene transfer. Science, 318, 1449-1452.

38. Kimelman,A., Levy,A., Sberro,H., Kidron,S., Leavitt,A., Amitai,G., YoderHimes,D.R., Wurtzel,O., Zhu,Y., Rubin,E.M., et al. (2012) A vast collection of microbial genes that are toxic to bacteria. Genome Res., 22, 802-809.

39. Li,W. and Godzik,A. (2006) Cd-hit: a fast program for clustering and comparing large sets of protein or nucleotide sequences. Bioinformatics, 22, 1658-1659.

40. Good,I.J. (1956) Some terminology and notation in information theory. Proceedings of the IEE Part C: Monographs, 103, 200. 
41. Zhang,Z. (2018) Improved Adam Optimizer for Deep Neural Networks. In 2018 IEEE/ACM 26th International Symposium on Quality of Service (IWQoS).pp. 1-2.

42. Gers,F.A., Schmidhuber,J. and Cummins,F. (2000) Learning to forget: continual prediction with LSTM. Neural Comput., 12, 2451-2471.

43. Keras,K. (2019) Deep learning library for theano and tensorflow. 2015.

44. Abadi,M., Isard,M. and Murray,D.G. (2017) A computational model for TensorFlow: an introduction. In Proceedings of the 1st ACM SIGPLAN International Workshop on Machine Learning and Programming Languages, MAPL 2017. Association for Computing Machinery, New York, NY, USA, pp. 1-7.

45. Koren,S., Walenz,B.P., Berlin,K., Miller,J.R., Bergman,N.H. and Phillippy,A.M. (2017) Canu: scalable and accurate long-read assembly via adaptive k-mer weighting and repeat separation. Genome Res., 27, 722-736.

46. Shafin,K., Pesout,T., Lorig-Roach,R., Haukness,M., Olsen,H.E., Bosworth,C., Armstrong,J., Tigyi,K., Maurer,N., Koren,S., et al. (2020) Nanopore sequencing and the Shasta toolkit enable efficient de novo assembly of eleven human genomes. Nat. Biotechnol., 38, 1044-1053.

47. Zulkower,V. and Rosser,S. (2020) DNA Features Viewer: a sequence annotation formatting and plotting library for Python. Bioinformatics, 36, 4350-4352.

48. Markowitz,V.M., Chen,I.-M.A., Palaniappan,K., Chu,K., Szeto,E., Grechkin,Y., Ratner,A., Jacob,B., Huang,J., Williams,P., et al. (2012) IMG: the Integrated Microbial Genomes database and comparative analysis system. Nucleic Acids Res., 40, D115-22.

49. Chen,I.-M.A., Chu,K., Palaniappan,K., Pillay,M., Ratner,A., Huang,J., Huntemann,M., Varghese,N., White,J.R., Seshadri,R., et al. (2019) IMG/M v.5.0: an integrated data management and comparative analysis system for microbial genomes and microbiomes. Nucleic Acids Res., 47, D666-D677.

50. Daligault,H.E., Davenport,K.W., Minogue,T.D., Bishop-Lilly,K.A., Broomall,S.M., Bruce,D.C., Chain,P.S., Coyne,S.R., Frey,K.G., Gibbons,H.S., et al. (2014) WholeGenome Yersinia sp. Assemblies from 10 Diverse Strains. Genome Announc., 2.

51. Wrobel,A., Ottoni,C., Leo,J.C. and Linke,D. (2018) pYR4 From a Norwegian Isolate of Yersinia ruckeri Is a Putative Virulence Plasmid Encoding Both a Type IV Pilus and a Type IV Secretion System. Front. Cell. Infect. Microbiol., 8, 373.

52. Carter,M.Q., Chen,J. and Lory,S. (2010) The Pseudomonas aeruginosa pathogenicity island PAPI-1 is transferred via a novel type IV pilus. J. Bacteriol., 192, 3249-3258.

53. Wallden,K., Rivera-Calzada,A. and Waksman,G. (2010) Type IV secretion systems: 
versatility and diversity in function. Cell. Microbiol., 12, 1203-1212.

54. Harms,A., Brodersen,D.E., Mitarai,N. and Gerdes,K. (2018) Toxins, Targets, and Triggers: An Overview of Toxin-Antitoxin Biology. Mol. Cell, 70, 768-784. 